\title{
Electron scattering effects at physisorbed hydrogen molecules on break-junction electrodes and nanowires formation in hydrogen environment
}

\author{
M. van der Maas ${ }^{1}$, S. Vasnyov ${ }^{1}$, B.L.M. Hendriksen ${ }^{1}$, O.I. Shklyarevskii ${ }^{1,2}$, and S. Speller ${ }^{1}$ \\ ${ }^{1}$ Institute for Molecules and Materials, Radboud University of Nijmegen \\ Toernooiveld 1, 6525 ED Nijmegen, the Netherlands \\ ${ }^{2}$ B. Verkin Institute for Low Temperature Physics and Engineering of the National Academy of Sciences of Ukraine \\ 47 Lenin Ave., Kharkov 61103, Ukraine \\ E-mail: shklyarevskii@ilt.kharkov.ua
}

Received January 17, 2012

\begin{abstract}
The physisorption of hydrogen molecules on the surface of gold and other coinage metals was studied using distance tunneling spectroscopy. We observed strong $N$-shaped deviation of the tunnel current (resistance) vs distance dependences from the exponential one. Such deviations are difficult to explain in the framework of the Tersoff-Hamann approximation. We suggest the scattering of the tunneling electrons by $\mathrm{H}_{2}$ molecules as origin for observed effect. We found that this phenomenon is also common for strongly adsorbed organic molecules with one anchoring group. Pulling $\mathrm{Au}, \mathrm{Cu}$ and $\mathrm{Pt}$ nanowires at $22 \mathrm{~K}$ in hydrogen environment show that the electrodes of break junctions are still connected up to very low conductances of $10^{-4}-10^{-6} G_{0}$ through hydrogen-metal one-atom chains.
\end{abstract}

PACS: 73.40.Jn Metal-to-metal contacts;

61.46. $+\mathbf{w}$ Structure of nanoscale materials;

68.65.La Quantum wires (patterned in quantum wells).

Keywords: nanowires, distance tunneling spectroscopy.

\section{Introduction}

The idea to build electronic devices based on single molecules dates back to 1974 [1] when the concept of a molecular rectifier was put forward. However, realistic experiments on conductance through a single molecule placed between metallic electrodes started 25 years later, when the modern techniques were able to produce stable atomicsized contacts. Those techniques include the Mechanically Controllable Break-Junction (MCBJ) method, which is widely used in studies of the molecular junction conductance (see reviews [2-4] and references therein).

It is not surprising that the behavior of the hydrogen molecule between the electrodes of MCBJ was studied very extensively due to the simplicity of the object. Most of the experiments were performed using transition metals. On many occasions the interaction of hydrogen with the surface of those metals is accompanied by molecular dissociation, even at low temperatures, and is highly material specific. Conductances of approximately one quantum unit $G_{0}=2 e^{2} / h \quad\left(1 / G_{0} \approx 12.9 \mathrm{k} \Omega\right)$ through the hydrogen bridge between Pt electrodes were reported in [5]. In case of Pt (and most probably for ferromagnetic metals [6]) the hydrogen atoms only can be found on the surface and the hydrogen bridge retains some of the properties of the $\mathrm{H}_{2}$ molecule $[5,7,8]$. For Pd [9], however, the underlayer of hydrogen distinctly changes the conductance through the hydrogen bridge. For W, Mo and Ta the dissociation of $\mathrm{H}_{2}$ molecules is quickly followed by dissolving of the hydrogen atoms (protons) into the electrodes via quantum diffusion. This effect drastically changes the conductance properties of the electrodes and eventually makes measurements of the contact conductance practically impossible $[10,11]$.

For gold the strong interaction between adsorbed molecules of hydrogen result in perceptible changes in the individual conductance traces measured at disconnection of electrodes and therefore in conductance histograms. This includes the appearance of the additional "fractional" peaks when measured in hydrogen environment [12] and incorporation of the hydrogen molecules ("hydrogen clamp") in the single-atom chains. The latter effect results in reduction of the chain conductivity and emerging of periodic structure in the conductance curves [13]. 
The reason for the observed effect is the strong reactivity of the gold nanowires predicted in $[14,15]$. The strong interaction between gold nanoclusters and hydrogen was reported in [16] and the potential applications of gold nanostuctures as catalysts, biochemical sensors etc. are widely discussed in [17].

In this article we report measurements of the conductance effects of a physically adsorbed hydrogen molecule in a MCBJ. This effect can be detected with the distance tunneling spectroscopy (DTS) method by measuring the contact conductance as a function of the gap $z$ between the electrodes [18]. The first experiments related to DTS were done on physically adsorbed He [19] and the deviation of the tunnel conductance $G(z)$ from exponential behavior was attributed to the strong reduction of the electron density of states close to the Fermi level by adsorbed atoms of helium predicted by Lang [20]. The same argument is given in scanning tunneling hydrogen microscopy (STHM) contrast of a gold dimer on a gold surface [21], where on top of the dimer (smaller tunneling gap) the conductance is lower than on the edge. Weiss et al., argue that this is caused by the depletion of the local density of states due to the Pauli repulsion between electrons in the metal and the electrons of the deuterium molecule in the gap. On the other hand the oscillating behavior of the tunnel current $\lg I(z) \quad\left(\lg I(z) \equiv \log _{10} I(z)\right)$ while moving the STM tip in the direction perpendicular to the adsorbed layers of $\mathrm{H}_{2} \mathrm{O}$ was explained by variation of the tunnel barrier height $\varphi$ in a rather wide range between 0.8 and $2.4 \mathrm{eV}$ [22]. Both explanations were consistent with the Tersoff-Hamann (TH) approximation [23]. Some situations, however, e.g., heterogeneous subnanoscale environment could neeed approaches beyond the scope of TH approach.

The data we are presenting here seem unlikely to be explained by a straightforward $\mathrm{TH}$ approach, as this would mean a increase of the barrier height to roughly $10 \mathrm{eV}$, or a depletion of the density of states of about 2 orders of magnitude. We propose a simple model on the basis of electron scattering by physically adsorbed $\mathrm{H}_{2}$ molecules that might explain the observed effects.

Additionally we found that the similar dependences of the tunnel conductance $G(z)$ are characteristic for organic molecules with a single anchoring group.

In the direct contact regime pulling characteristics for $\mathrm{Au}, \mathrm{Cu}$ and $\mathrm{Pt}$ are strikingly different from those observed for $\mathrm{Au}$ in [13].

\section{Experiment}

In our experiments we used a standard MCBJ technique described elsewhere [24]. All measurements were done either in a cryogenic ultra-high vacuum or in the atmosphere of ultra-pure $(99,9999 \%)$ hydrogen. The vacuum pot with the sample was cooled by pumping of He vapor through a special transfer tube with regulated input, in a way similar to that in the flow cryostat. In spite of the relatively low absolute accuracy of Allen-Bradley carbon resistor thermometer we used for the temperature readings (the error of the calibration curve at the temperatures of $20 \mathrm{~K}$ can be as large as $\pm 0.2 \mathrm{~K}$ ) we were able to maintain the temperature of the sample within $\pm 0.01 \mathrm{~K}$ for sufficiently long time (up to 3-4 hours). Normally the vacuum pot was initially overcooled by $\approx 1-2 \mathrm{~K}$ and the desirable temperature was maintained with a heater, monitored by a Lake Shore 340 temperature controller. The thermal drift of the electrodes in the tunneling regime usually was less than 2-3 pm per minute.

All measurements presented below were done at $22 \mathrm{~K}$ (above the boiling point of hydrogen $20.268 \mathrm{~K}$ ). We found that the effects reported below are rather insensitive to the amount of hydrogen admitted into the vacuum pot and can be observed at hydrogen pressures (measured at the top of cryostat) ranging from $10^{-3}$ to 100 Torr.

\section{Results and discussion}

\subsection{Scattering of electrons by the hydrogen molecule}

The most spectacular curves of the contact conductance vs interelectrode separation $z$ measured in the quantum conductance units $G_{0}=2 e^{2} / h$ for Au MCBJ are presented in Fig. 1.

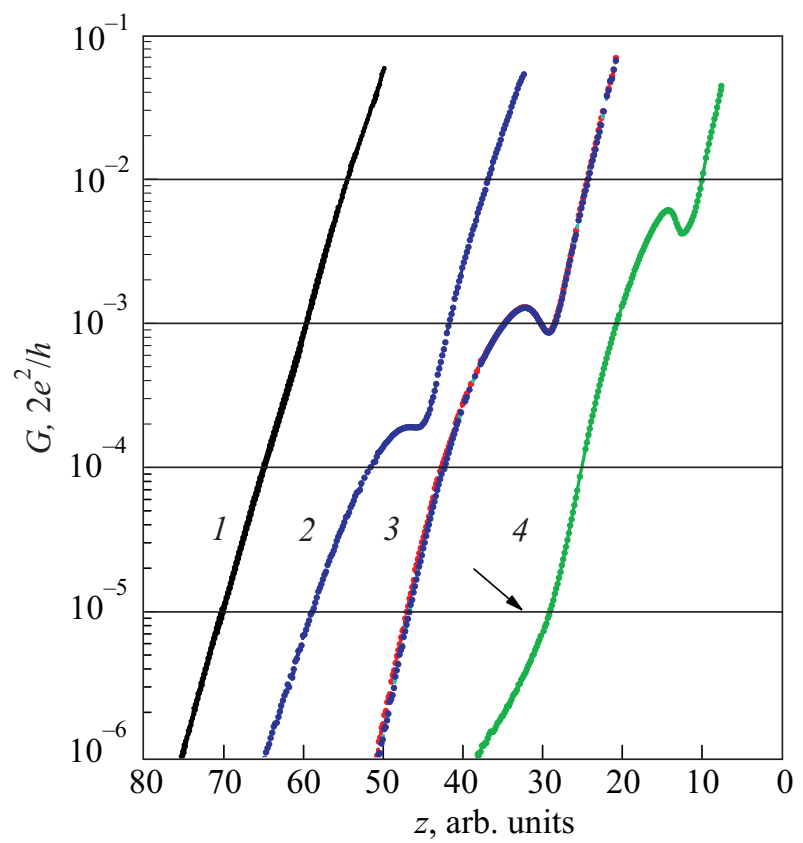

Fig. 1. Typical dependences of the contact conductance $G$ versus electrode separation $z$ for a Au MCBJ in vacuum (curve 1), and in case of physically adsorbed $\mathrm{H}_{2}$ (curves $2-4$ ). The last dependence shows the possible presence of a second layer of adsorbed hydrogen molecules (or the presence of a molecule adsorbed on the "sharp" electrode) at $G$ dependence around $10^{-5} G_{0}$ (indicated by the black arrow). 
Under cryogenic UHV condition this dependence demonstrates a perfect exponential behavior in the entire range of conductances (curve 1). Admitting even a small $(\sim 0.5 \mu \mathrm{M})$ amount of hydrogen results in a strong deviation of $G(z)$ from the exponential behavior. All curves presented in Fig. 1 were measured in the process of approaching the electrodes to each other up to the conductance less than $0.1 G_{0}$ (thereby avoiding the jump to direct contact due to metallic adhesion) and some of them also in the reverse process of subsequent withdrawal of the electrodes (curves 1,3). In most cases the resulting dependences are practically identical. Compared to our measurements for physisorbed He [19], the effect of adsorbed Hydrogen molecules on the tunnel conductance is much stronger. The tunnel current reaches its maximum and then decreases as the distance between electrodes continue to decrease (curves $2-4$ ). This singularity usually occurs in the range between $10^{-2}$ and $10^{-4} G_{0}$. The spread in the data is probably related to the variation in the position of the adsorption potential minimum for $\mathrm{H}_{2}$ at specific adsorbtion sites on the rough surface of the electrodes (in some respects similar to the top, hollow or bridge adsorbtion sites on the metallic surface). We assume that these $N$-shape signatures in the curves presented in Fig. 1 are corresponding to the situation when a hydrogen molecule adsorbed on the "blunt" electrode is located directly opposite (or slightly shifted) to the foremost atom of the "sharp" electrode. In other cases the distortion of the $\lg G(z)$ curves is less pronounced and is reduced to a "plateau". Figure 2 shows the transformation of those dependences due to the thermal drift of the electrodes (with a switched off feed-

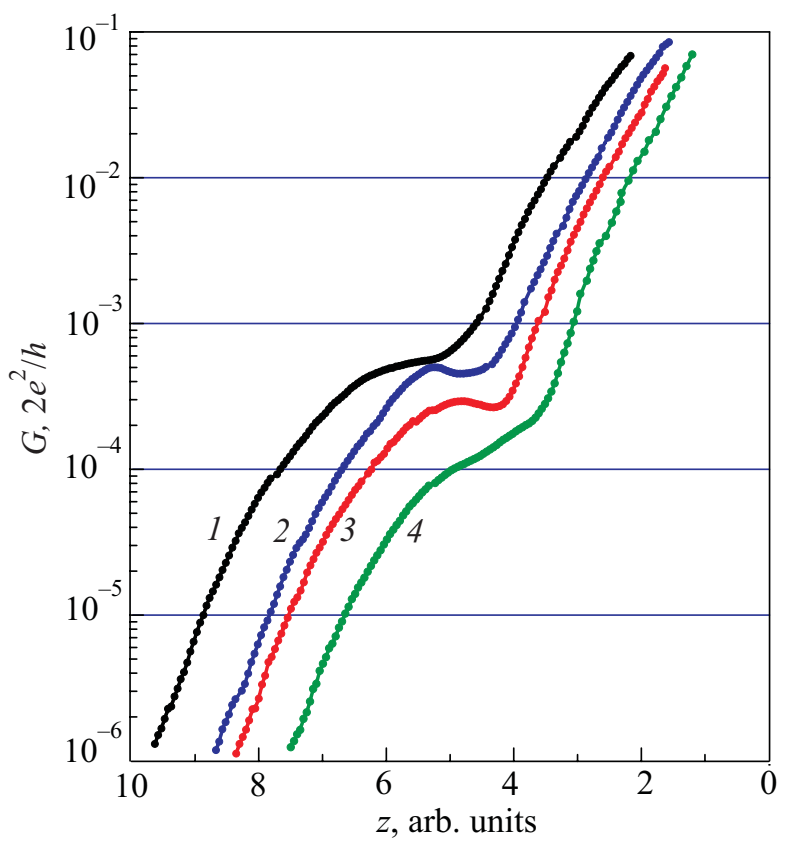

Fig. 2. Evolution (from left to right) of $G(z)$ dependences in the course of the thermal drift of the electrodes as the temperature drops from 22 to $21.7 \mathrm{~K}$ with feedback switched off. back circuit of the temperature controller) in $z$ and lateral directions. The estimated drift in the $z$ and/or lateral direction does not exceed $1-2 \AA$.

The bias dependence of the $\lg G(z)$ curves was observed before in the case of the physically adsorbed helium [19]. In the rather narrow range of $V_{b}(1-20 \mathrm{mV})$ the deviation from exponential behavior increased with the decrease of bias voltage. This effect could not be properly explained at that time. For adsorbed hydrogen molecules the influence of the $V_{b}$ on the shape of $\lg G(z)$ curves can be observed in the higher bias range up to $100 \mathrm{mV}$ (Fig. 3). At even higher bias voltages the contact conductance becomes unstable and shows a sudden jump in the contact conductance (see inset in Fig. 3). At $V_{b}>500 \mathrm{mV}$ any recording of $\lg G(z)$ is already practically impossible. Due to the relatively high polarizability of the hydrogen molecule the instability starts at the estimated field strength of $0.02-0.05 \mathrm{~V} / \AA$ whereas in the case of adsorbed He measurements at field strength as high as $0.5 \mathrm{~V} / \AA$ were still possible.

Measurements for $\mathrm{Ag}, \mathrm{Cu}$ and $\mathrm{Pt}$ show clear deviation of $G(z)$ dependences from exponential behavior. However this effect is less pronounced than in the case of Au. It should be remarked that the conductance value corresponding to the singularity in $\lg G(z)$ curves can not be viewed as a "conductance" through the hydrogen molecule, as in this case it does not form chemical bonds with the electrodes.

As stated above the $N$-shaped $\lg G(z)$ curves are not easily explained using the TH model. Otherwise one must accept either a reduction of electron DOS by 1-2 orders of magnitude or the sudden few fold increase of the tunnel

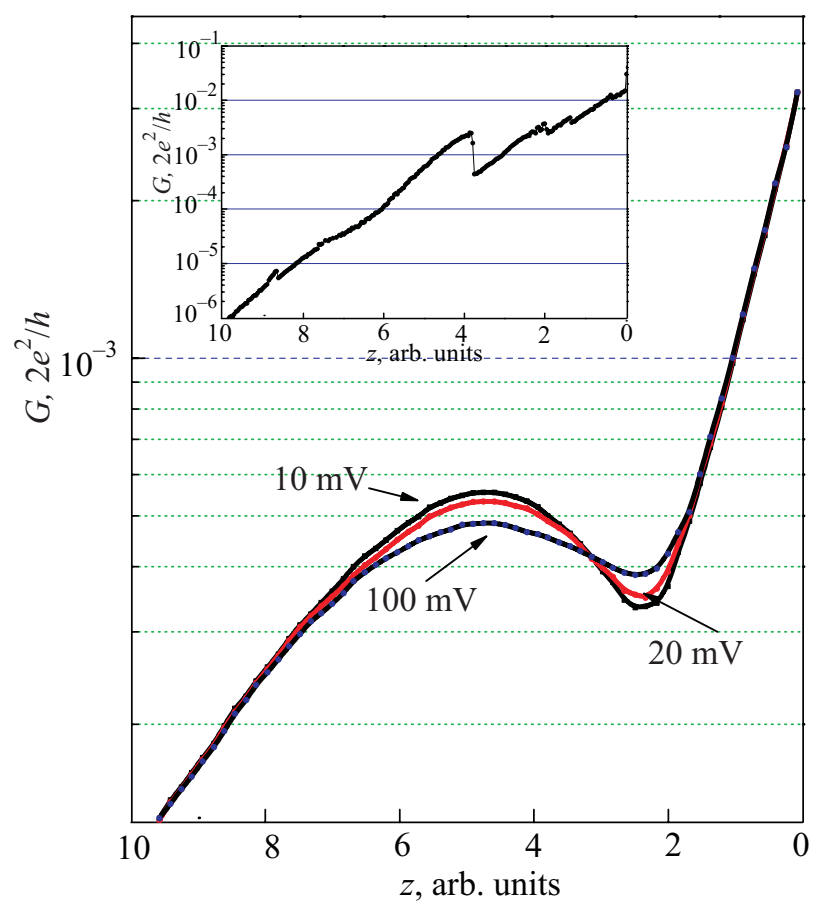

Fig. 3. Part of the $\lg G(z)$ dependence for the same junction at different bias voltages. Inset: instable $G(z)$ curve measured at $V_{b}=400 \mathrm{mV}$. 
barrier height $\varphi$. The above results suggest that the most plausible phenomenon for the effect observed might be the scattering of the tunneling electrons by a hydrogen molecule. At relatively large separations between the electrodes (5 to $7 \AA$ ) the scattering is relatively small. As the distance between the electrodes decreases, the percentage of tunneling electrons scattered by the impenetrable hydrogen molecule rapidly increases. At a certain moment this result in the reduction of the tunnel current until the moment the repulsive Pauli potential starts to expel the $\mathrm{H}_{2}$ molecule from the tunnel gap. In the next section we use a simple model to reproduce in a simulation the observed $N$-shape deviation described above.

\subsection{Simple simulations}

The exact description for the observed effects requires a quantum mechanical approach and rather complicated calculations. However, the main features of the $G(z)$ dependences can be described qualitatively using a very simple classical model presented in Fig. 4. This involves only geometrical considerations.

In this model we assume that one of the electrodes is atomically flat on the scale of a few interatomic distances while the second one is atomically sharp. The hydrogen molecule is represented by an impenetrable for electrons sphere of diameter $d$ at the distance $P \sim 2.5 \AA$ from the flat electrode (for the majority of metals the distance between the surface and the physisorbed molecule is $2.3-2.7 \AA$ [25]). The parameter $Z_{\text {push }}$ represents the distance at which the expelling of the molecule starts, as the separation between electrodes decreases, and is held at 4.5-5 $\AA$. The distance $Z_{\text {push }}$ of electrode 2 at which expelling of the hydrogen molecule from the interelectrode space begins is at the deepest point of the potential well in the adsorbtion potential curve. For $\mathrm{Au}, \mathrm{Ag}$ and $\mathrm{Cu}$ the well depth is approximately $30 \mathrm{meV}$ deep [26]. The expelling of the $\mathrm{H}_{2}$ molecule is done in a linear way, for every step electrode 1 takes towards electrode 2 the molecule is expelled a step along the plane of electrode 2 (i.e. perpendicular to the $z$-axis). We also suggest that only electrons in the cone

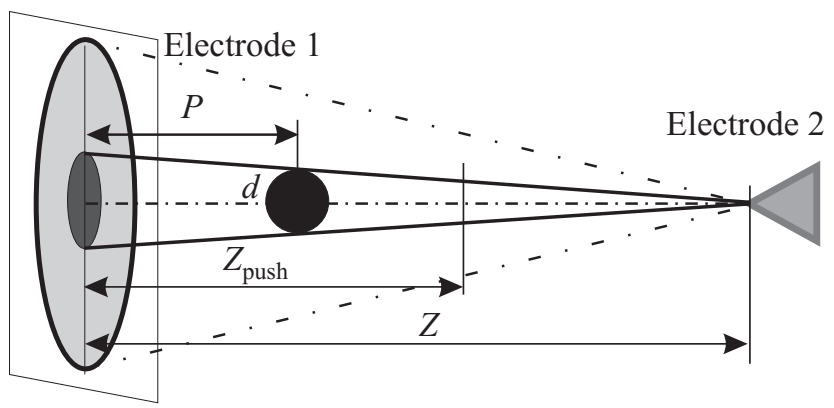

Fig. 4. Simple model for electron scattering on a $\mathrm{H}_{2}$ molecule. The shadowed area on electrode 2 represents the amount of backscattered electrons. with solid angle of 30 degrees are contributing to the conductance. As the distance between the two electrodes is decreased the amount of backscattering by the hydrogen molecule will increase, this is modeled by an increase in the "shadow" of the molecule on electrode 2 . Under above parameters the satisfactory agreement with experiment can be found assuming that effective scattering radius is $0.5-0.6 \AA$. For the diameter $d$ this gives 1.0-1.2 $\AA$ for $d$ and correlates with the small dimension of the hydrogen molecule (the distance between protons is $0.74 \AA$ ).

3D plots of the $G(z)$ dependences are presented in Fig. 5 as a function of the pushing distance $Z_{\text {push }}$ assuming scattering radius $R_{\text {scatt }}$ is equal to $0.55 \AA$ (Fig. 5,a) and as a function of scattering radius at $Z_{\text {push }}=4.8 \AA$ (Fig. 5,b). The simulated characteristics clearly reproduce the effect of conductance reduction observable in a relatively narrow range of $Z_{\text {push }}$ and $R_{\text {scatt }}$, respectively.

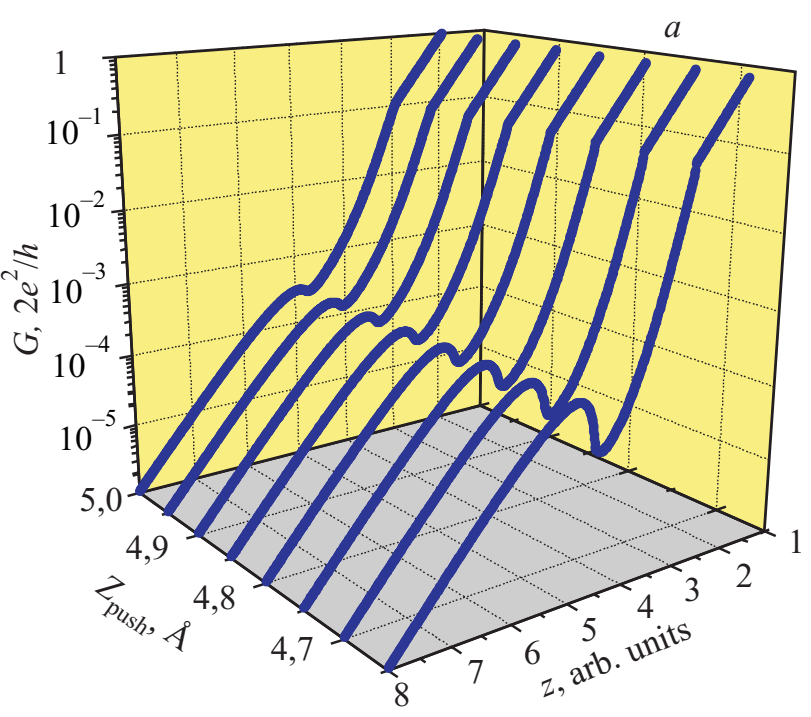

$b$

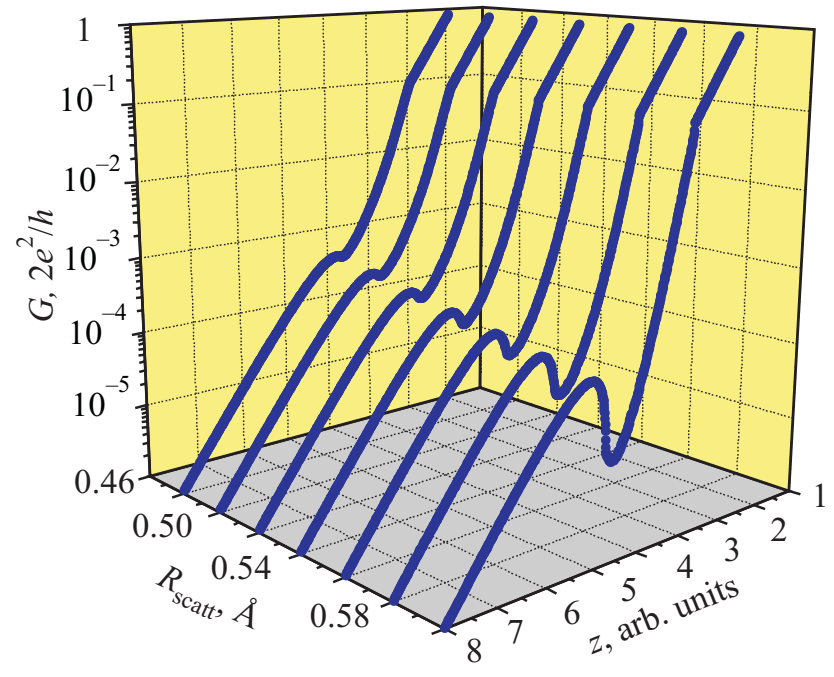

Fig. 5. 3D plots for the families of the $G(z)$ dependences as a function of the pushing distance $Z_{\text {push }}$ assuming scattering radius $R_{\text {scatt }}$ is equal $0.55 \AA(a)$ and as a function of scattering radius at $Z_{\text {push }}=4.8 \AA(b)$. 
In the case when the hydrogen molecule is shifted away from the contact axis the effect of conductance reduction disappears rather fast (curves 1 and 2 in Fig. 6). This correlates with the fact that experimental observation of this effect occurs only for $5-10 \%$ of all curves measured. The simple model of scattering shows qualitatively the same trend as observed in the data, however, is unable to explain the apparent increase of the tunneling gap (Fig. 1). This effect can be understood only if we suggest that some of the tunneling parameters in $\mathrm{TH}$ approximations are not constant and changing with $z$. It is a well known fact that the adsorbtion of hydrogen on the surface may reduce or increase the work function of the metal. This effect is included in curve 3 (increase) and 4 (reduction of the work function) in Fig. 6 after the molecule is pushed out.

The effect of electron scattering is not limited only to physically adsorbed hydrogen but bears universal character and can be observed on other sufficiently strongly adsorbed molecules. To prove it we studied conductance traces of 1-pentanethiol and thiophenol chemisorbed on the electrodes of gold MCBJ. These measurements were done at room temperature in 1,2,4-trichlorbenzene. (For experimental details of such measurements see Ref. 27.) We were able to detect the presence of 1-pentanethiol and thiophenol at concentration as low as 10 nanomoles. The typical individual conductance curves show $N$-shaped behavior and contribute to the pronounceable peak in conductance histogram presented in the lower panel of Fig. 7. However, the position of the anomaly on individual conductance curves, and therefore position of the maximum in the conductance histogram, may change within 1 to 2 orders of magnitude. Therefore, it is impossible to identify the molecules by a single conductance value. However, the measurement is sensitive for the detection of molecules on the surface, by identification of an $N$-shape in the conductance curves.

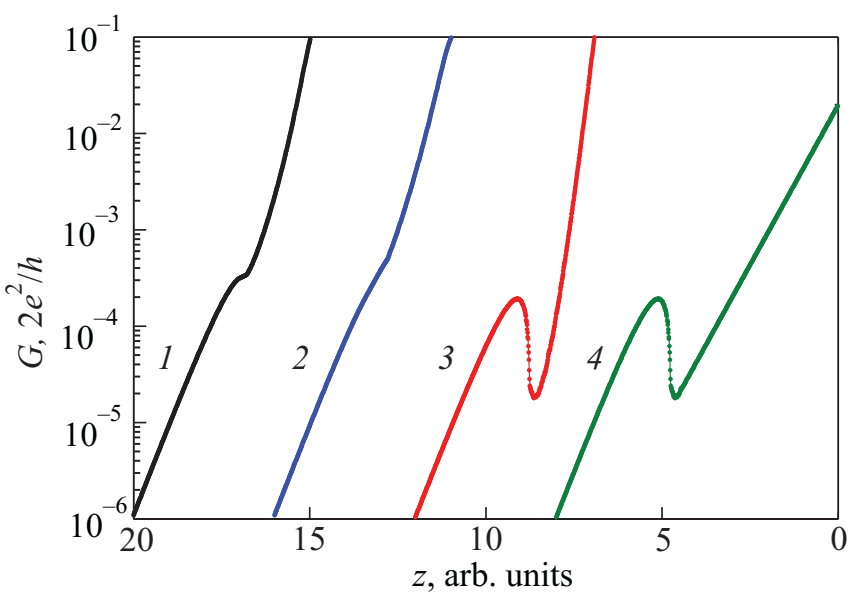

Fig. 6. Conductance curves 1 and 2 are simulated for the hydrogen molecule shifted from the contact axis by 0.1 and $0.2 \AA$. Curves 3 and 4 correspond to increase and reduction of the metal work function after expelling the hydrogen molecule from the interelectrode space.

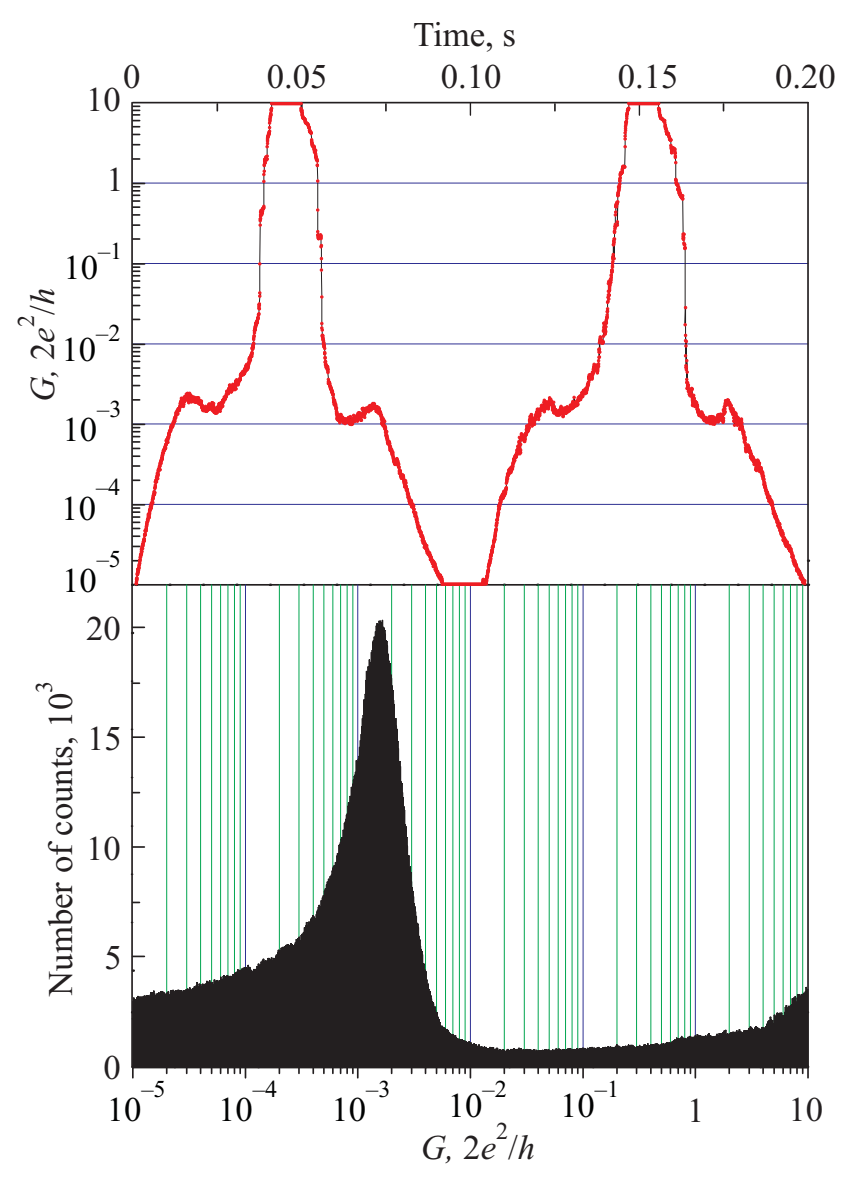

Fig. 7. Conductance traces for adsorbed thiophenol molecules, measured with a $10 \mathrm{~Hz}$ triangle ramp piezovoltage $(a)$. Conductance histogram constructed from 2000 individual traces $(b)$.

\subsection{Pulling the nanowires in molecular hydrogen environment}

All measurements for hydrogen molecules presented above were done in the tunneling regime without bringing electrodes in direct mechanical and electrical contact. In this section we present conductance traces measured in the course of pulling electrodes, starting from direct contact with conductances of $10-20 G_{0}$. It was shown in [13] that a hydrogen molecule can be included in a single-atom nanowire clamping to neighboring gold atoms. At temperatures starting from $4.2 \mathrm{~K}$ and below $10-15 \mathrm{~K}$ our results for pulling gold nanowires coincide nicely with these data. The distance between the electrodes was calibrated using either $G(z)$ dependences measured in UHV or the linear part of $G(z)$ curves measured in a hydrogen environment. Assuming that the work function values for $\mathrm{Cu}, \mathrm{Au}$ and $\mathrm{Pt}$ are between 4.5 and $5.5 \mathrm{eV}$ we can safely use a coefficient as $(1 \pm 0.1) \AA$ A per decade of conductance decay for calibration.

However, at higher temperatures (and enhanced amount of hydrogen) the behavior of the conductance curves changes dramatically. Moreover, hydrogen-metal conducting chains can be pulled from $\mathrm{Cu}$ that under UHV condi- 
tions do not form monatomic chains. Like in the measurements presented above the temperature was $22 \mathrm{~K}$ and the pressure of hydrogen 50 Torr. It should be noted also that the pulling speed in most of the experiments was usually less than $1 \mathrm{~nm} / \mathrm{min}$.

A typical pulling curve for gold is presented in Fig. 8, $a$ and shows that the electrodes stay connected at distances as large as $\sim 1.5 \mathrm{~nm}$ or approximately 5 interatomic distances. The conductance of the atomic chain drops as low as $10^{-5} G_{0}$, or more than 3 order of magnitude below the conductances reported in [13]. Like in [13] the conductance decreases in a nonmonotonic way, with sudden drops and subsequent recovery sometimes even to higher values of $G$. This effect was explained in [13] by the assumption that the conductance through the hydrogen molecules stretched parallel to the contact axis is higher than for molecules oriented perpendicular to it. This assumption is based on simulations [15] where the conductance through a gold dimer welded by a hydrogen molecule was calculated. The rich structure of $G(z)$ curves in our experiments and the low conductance of the atomic chain indicate that more than one $\mathrm{H}_{2}$ molecule is incorporated into the nanowire in different configurations including probably "hydrogen wire" configurations with conductances as low as $0.01 G_{0}$. This is possibly mainly due to the elevated temperature of the experiment and high hydrogen pressure that ensures high mobility and immediate availability of $\mathrm{H}_{2}$ molecules

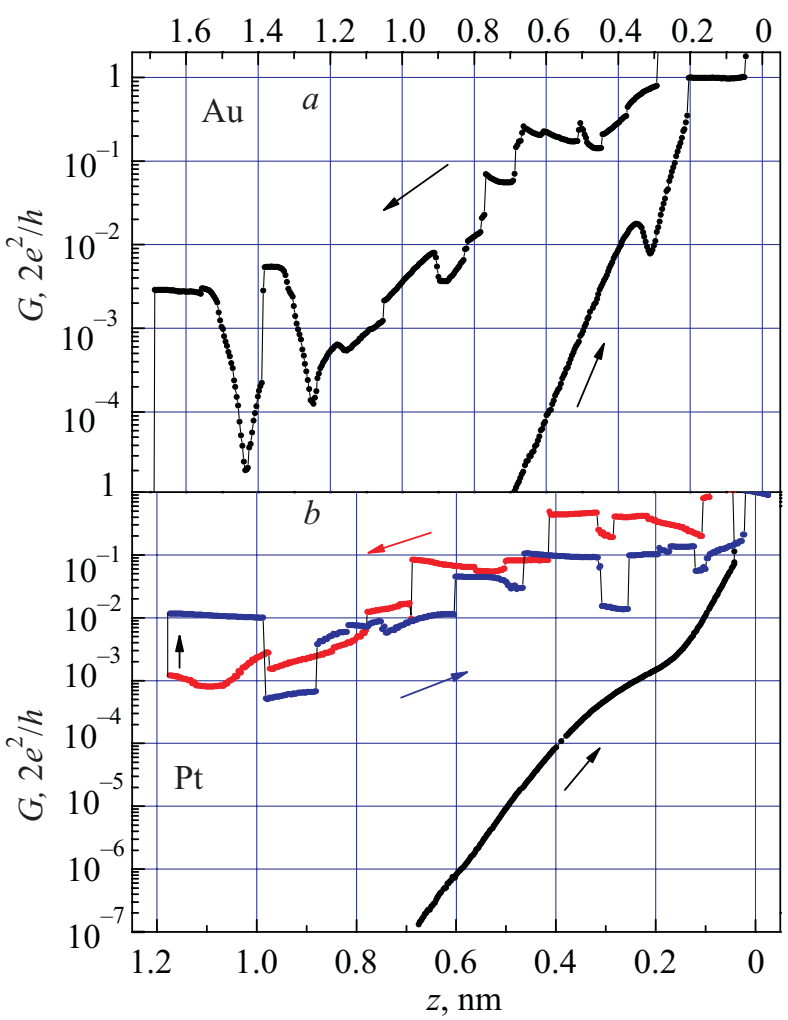

Fig. 8. Typical conductance curve for a gold MCBJ, on approach to the direct contact and, subsequent pulling of the nanowire $(a)$. Pulling curves for Pt $(b)$. along the atomic chain. It is also likely that the low pulling speed also contributes to the observed effects. On rare occasion conductance curves demonstrated quasiperiodic structure although lack of reliable statistics prevent us from drawing parallels with observation reported in [13].

We found that the "hydrogen clamp" is also working for some other materials. Not surprisingly it occurs for silver which is known for the possibility to pull short chains at cryogenic UHV condition and long chains in the presence of oxygen [28]. The conductance curves for silver are similar to those of gold.

Platinum also forms single atom chains under UHV conditions [29]. Evidence that hydrogen molecules can be incorporated in the mono-atomic chains of $\mathrm{Pt}$ atoms was presented in [30]. Like in [13] these measurements were done at $4.2 \mathrm{~K}$ and in the limited range of conductances below $G_{0}$. In our case the $G(z)$ pulling curves for Pt are looking somewhat different (Fig. 8,b). They consist mainly of flat or slightly tilted plateaus interchanged with the abrupt jumps in conductance (up and down) of approximately one order of magnitude. This difference in conductance behavior can be explained by the fact that at $22 \mathrm{~K}$ dissociation of hydrogen molecules is quite probable and a hydrogen atom (proton) can be incorporated into the single atom chain. For gold such a situation results in a so-called "Hydrogen Switch" reducing conductance of the wire to zero [15]. For Pt conductance through the single atom is still possible and most probably has little or no dependence on stretching (in contrast to the hydrogen molecule). That explains the flatness of the plateaus in the conductance curves (Fig. 8,b). The transition from pulling the Pt wire to its compression, usually is accompanied by a immediate jump in conductance at the return point, but as we continue to compress the nanowire the character of the $G(z)$ dependence remains generally the same, until the final transition to direct metallic contact. Note that the "pulling" distance and the "return distance" are practically the same and therefore the process is completely reversible.

For copper (in contrast to gold, silver, platinum or iridium) single atom chains were never observed under UHV conditions, although according to [28] such an effect is possible under an oxygen atmosphere at elevated temperatures. Fractional conductance for gold and copper was observed for nanocontacts in solution under electrochemical potential control in the range of hydrogen evolution [31]. Theoretical analysis performed in [32] demonstrated that a $\mathrm{Cu}$ nanowire can be rather reactive to hydrogen. In our experiments we observed two distinct types of copper nanowire behavior.

Part of the contacts demonstrated pulling curves that were also characteristic both for $\mathrm{Au}$ and $\mathrm{Ag}$ with an unavoidable break at conductances of $10^{-3}-10^{-4} G_{0}$ (see Fig. 9,a). For other curves the final break to zero conductance did not occur until $G \sim 10^{-6} G_{0}$ (Fig. 9,b), and for the larger part the contact conductance decreases in a gra- 


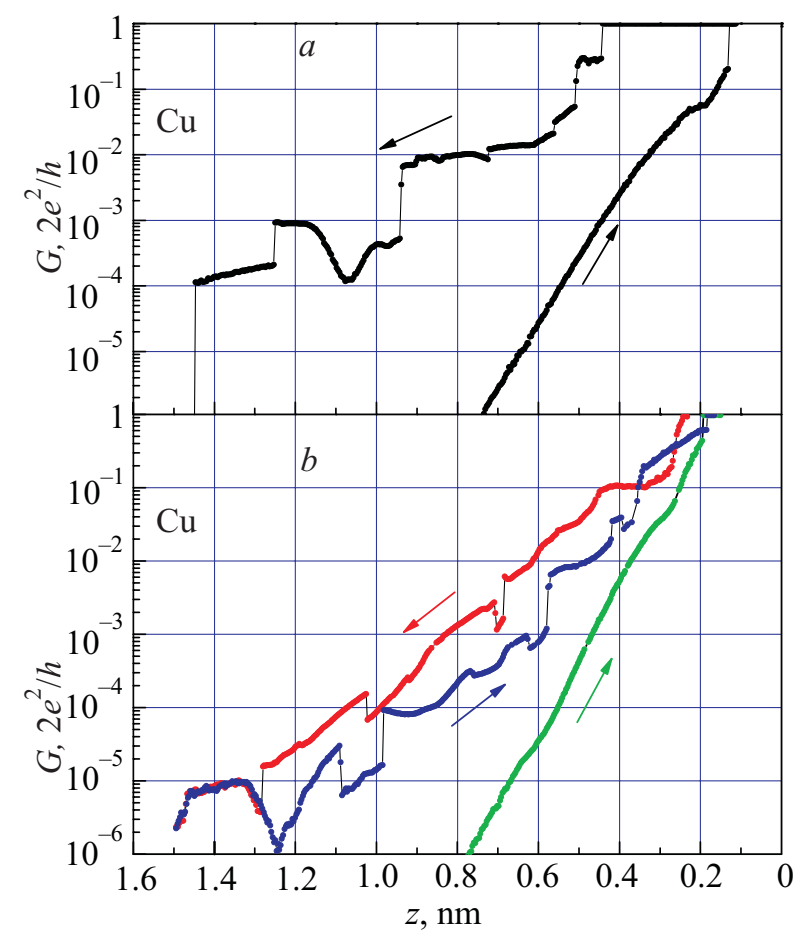

Fig. 9. Two types of conductance curves for copper MCBJ on approach to direct contact and subsequent pulling of the nanowire.

dual way. The difference between these two types of dependences might very well be related to the crystallographic orientations of the electrodes. It is also possible that hydrogen molecules can be incorporated in other parts of the surface of the electrodes besides the single atom chain.

Concluding, we found that the deviation of $\lg G(z)$ curves from exponential behavior in the presence of physically adsorbed hydrogen at the surface of electrodes of MCBJ are not easily explained in the framework of Tersoff-Hamann approximation by variation of electron DOS in the electrodes or variation of the tunnel barrier height $\varphi$. We propose that a plausible model for the observed effects is backscattering of tunneling electrons by the hydrogen molecule.

Part of this work was supported by the Nanotechnology network in the Netherlands NanoNed and the Stichting voor Fundamenteel Onderzoek der Materie (FOM) which is financially supported by the Nederlandse Organisatie voor Wetenschappelijk Onderzoek (NWO). O.I.S. wishes to thank the FOM and NWO for a visitor's grant.

1. A. Aviram and M.A. Ratner, Chem. Phys. Lett. 29, 277 (1974).

2. R.L. McCreery, Chem. Mater. 16, 4477(2004).

3. F. Chen, J. Hihath, Z.F Huang, X.L. Li, and N.J. Tao, Annu. Rev. Phys. Chem. 58, 535 (2007).

4. N.J. Tao, Nature Nanotechnology 1, 173 (2006).

5. R.H.M. Smit, Y. Noat, C. Untiedt, N.D. Lang, M.C. van Hemert, and J.M. van Ruitenbeek, Nature 419, 906 (2002).
6. C. Untiedt, D.M.T. Dekker, D. Djukic, and J.M. van Ruitenbeek, Phys. Rev. B69, 081401(R) (2004).

7. D. Djukic, K.S. Thygesen, C. Untiedt, R.H.M. Smit, K.W. Jacobsen, J.M. van Ruitenbeek, Phys. Rev. B71, 161402 (2005).

8. K.S. Thygesen and K.W. Jacobsen, Phys. Rev. Lett. 94, 036807 (2005).

9. Sz. Csonka, A. Halbritter, G. Mihaly, E. Jurdik, O.I. Shklyarevskii, S. Speller, and H. van Kempen, Phys. Rev. Lett. 93, 016802 (2004).

10. D. den Boer, O.I. Shklyarevskii, J.A.A.W. Elemans, and S. Speller, J. Phys. Conf. Ser. 61, 239 (2007).

11. D. den Boer, O.I. Shklyarevskii, J.A.A.W. Elemans, and S. Speller, Phys. Rev. B77, 165423 (2008).

12. Sz. Csonka, A. Halbritter, G. Mihaly, O.I. Shklyarevskii, S. Speller, and H. van Kempen, Phys. Rev. Lett. 93, 016802 (2004).

13. Sz. Csonka, A. Halbritter, and G. Mihaly, Phys. Rev. B73, 075405 (2006).

14. S.R. Bahn, N. Lopez, J.K. Norskov, and K.W. Jacobsen, Phys. Rev. B66, 081405(R) (2002).

15. R.N. Barnett, H. Hakkinen, A.G. Scherbakov, and U. Landman, Nano Lett. 4, 18452004.

16. H.J. Zhai, B. Kiran, and L.S. Wang, J. Chem. Phys. 121, 8231 (2004).

17. G.J. Hutchings, M. Brust and H. Schmidbaur, Chem. Soc. Rev. 37, 1759 (2008).

18. J. Halbritter, G. Repphun, S. Vinzelberg, G. Staikov, and W.J. Lorenz, Elrctrochimica Acta 40, 1394 (1995).

19. R.J.P. Keijsers, J. Voets, O.I. Shklyarevskii, and H. van Kempen, Phys. Rev. Lett. 76, 1138 (1996).

20. N.D. Lang, Phys. Rev. Lett. 56, 1164 (1986).

21. C. Weiss, C. Wagner, C. Kleimann, M. Rohlfing, F.S. Tautz, and R. Temirov, Phys. Rev. Lett. 105, 086103 (2010).

22. M. Hugelmann and W. Schindler, Surf. Sci. 541, L643 (2003).

23. J. Tersoff and D.R. Hamann, Phys. Rev. Lett. 50, 1998 (1983).

24. N. Agrait, A. Levy Yeyati, and J.M. van Ruitenbeek, Phys. Reports. 377, 8103 (2003).

25. C.M. Brown, Yun Liu, and D.A. Neumann, Pramana-J. Phys. 71755 (2008).

26. S. Andersson, Z. Wilzén, and M. Persson, Phys. Rev. B38, 2976 (1988).

27. D. den Boer, M.J.J. Coenen, M. van der Maas, T.P.J. Peters, O.I. Shklyarevskii, J.A.A.W. Elemans, A.E. Rowan, and S. Speller, J. Phys. Chem. C113, 15412 (2009).

28. W.H.A. Thijssen, D. Marjenburgh, R.H. Bremmer, and J.M. van Ruitenbeek, Phys. Rev. Lett. 96, 026806 (2006).

29. R.H.M. Smit, PhD Thesis, Universiteit Leiden (2003).

30. M. Kiguchi, R. Stadler, I.S. Kristensen, D. Djukic, and J.M. van Ruitenbeek, Phys. Rev. Lett. 98, 146802 (2007).

31. M. Kiguchi, T. Konishi, S. Miura, and K. Murakoshi, Nonotechnology 18, 424011 (2007).

32. E. Santos, P. Quaino, G. Soldano, and W. Schmickler, Electrochem. Commun. 11, 1764 (2009). 